\title{
INTEGRANDO NATURALEZA Y PASADO HISTÓRICO: EL SITIO ARQUEOLÓGICO CAPILLA DE SAN ALONSO
}

Mario L. CHATELLENAZ ${ }^{1}$ y Tamara YUNES NÚÑEZ ${ }^{2}$

Palabras clave: sitio arqueológico, ruinas jesuíticas, San Alonso, biodiversidad, Corrientes.

Resumen: El Sitio Arqueológico $\mathrm{Ca}^{-}$ pilla de San Alonso (Departamento Santo Tomé, Corrientes) posee ruinas jesuíticas que datan del siglo XVIII, por lo que ha sido considerado Patrimonio Cultural, Natural y Turístico. Las ruinas están rodeadas por un remanente de Bosque Paranaense que constituye un atractivo adicional para los turistas que visitan el Sitio. Con el objetivo de vincular los conceptos culturales y arqueológicos del sitio con la riqueza de su medio biofísico, se realizaron relevamientos de la vegetación, y de aves y mamíferos, entre mayo y julio de 2015. Se registraron 44 especies de plantas vasculares, 65 de aves y ocho de mamíferos. Aunque estos números no son definitivos, y falta información referida a otros grupos de organismos, confirman la importancia del bosque remanente en el Sitio como patrimonio natural y atractivo turístico, y su potencial para realización de actividades de investigación y educación ambiental.

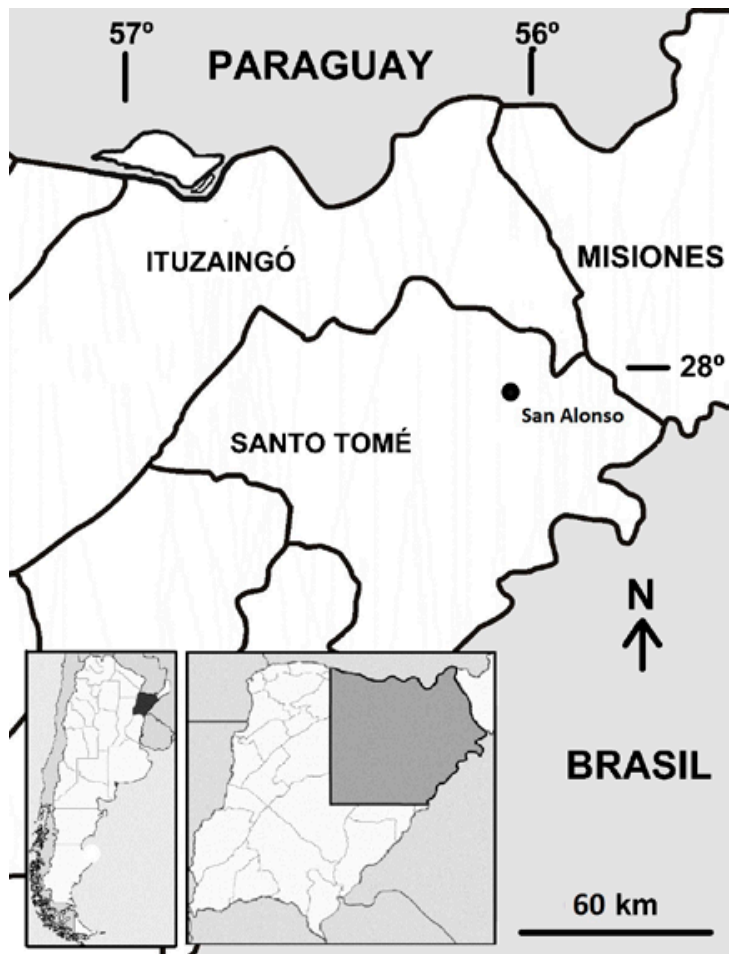

Fig. 1. Ubicación geográfica del Sitio Arqueológico Capilla de San Alonso.

\section{INTRODUCCIÓN}

El Sitio Arqueológico Capilla de San Alonso se encuentra situado a $13 \mathrm{~km}$ al nordeste de la ciudad de Gobernador Virasoro, en el departamento Santo Tomé,

(1) Licenciado en Zoología. Laboratorio de Ornitología y Mastozoología. Facultad de Ciencias Exactas y Naturales y Agrimensura, Universidad Nacional del Nordeste. Av. Libertad 5470 (3400). Corrientes, Argentina. Correo electrónico: mlchatellenaz@gmail.com

(2) Ingeniera Ambiental y Magister en Gestión del Agua. Establecimiento Las Marías, C. 25. Ruta Nacional 14, Km739, Gobernador Virasoro (3342). Corrientes, Argentina. Correo electrónico: tamarapoblet@yahoo.com.ar 


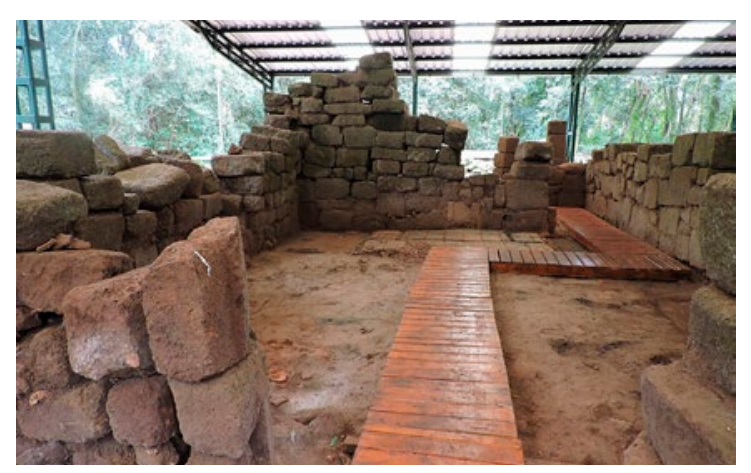

Fig. 2. Aspecto parcial de las ruinas de la antigua capilla jesuítica.

provincia de Corrientes (Fig. 1). Tiene una superficie de 4,3 ha, ubicadas en terrenos de propiedad de la Empresa Forestal Bosques del Plata S.A., con quien la Municipalidad de Gobernador Virasoro ha firmado convenios para la ejecución de actividades turísticas y de investigación.

El conjunto formado por la Capi1la Jesuítica Guaraní (Fig. 2), el Cementerio Rural y su entorna natural formado por bosque nativo fue declarado Patrimonio Cultural, Natural y Turístico por Ordenanza 771/2015 del Honorable Concejo Deliberante de la ciudad de Gobernador Virasoro. En dicha Ordenanza se destaca el valor de la diversidad biológica del bosque presente en el sitio, y se realza su valor para la educación ambiental. Según esta Ordenanza, el Sitio actuaría como "nodo de comunicación entre la Reserva Esteros del Iberá, la Microrregión de la Yerba Mate y Tierra Roja, Asociación Ruta de la Yerba Mate, el Corredor Jesuítico Guaraní de Corrientes, las Misiones Jesuíticas Patrimonio de la Humanidad (UNESCO) de la Provincia de Misiones y el circuito de los 30 pueblos jesuíticos guaraníes ubicados entre Argentina, Paraguay, Brasil y Uruguay".
Desde el año 2012, la Municipalidad de Gobernador Virasoro ha ejecutado el "Proyecto de Puesta en Valor Cultural y Turística de la Capilla Jesuítica Guaraní de San Alonso" referido principalmente a la realización de excavaciones arqueológicas y acceso controlado de visitantes al Sitio. No obstante, el mismo no contemplaba los aspectos naturales del Sitio, de gran atractivo para los visitantes. En consecuencia, se propuso la realización de un proyecto que se enfocara en dichos aspectos. Este proyecto, denominado "Interacción con el Paisaje $\mathrm{Na}^{-}$ tural del Sitio Arqueológico del Paraje San Alonso", tuvo como propósito: a) realizar un relevamiento de flora y fauna del sitio; b) vincular los conceptos culturales y arqueológicos del sitio con la riqueza de su medio biofísico. En este trabajo se exponen los resultados obtenidos. Se espera que los mismos sirvan para el desarrollo de una estrategia de turismo coherente y realista, ajustada al potencial del área.

\section{LA NATURALEZA DEL ÁREA Relieve y suelo}

El área donde se encuentra el Sitio está comprendida en una unidad geomorfológica denominada "Planicie CorrentinoMisionera del Sector Oriental”, una de las que integran la Llanura Mesopotámica. E1 relieve, suavemente ondulado, se caracteriza por la presencia de lomadas y colinas de entre 120 y 130 msnm separadas por pequeños valles, muchos de ellos correspondientes a arroyos, y la presencia de basaltos y areniscas situados a poca profundidad (Popolizio 1989). Estos basaltos, conocidos como 
"basaltos de la Serra Geral", deben su origen a las sucesivas coladas de lava que tuvieron su centro efusivo en el actual territorio brasileño y cubrieron toda la región durante el Jurásico y el Cretácico inferior y medio (Teruggi 1970). La alteración profunda del basalto por vientos, lluvias y temperatura, dio origen a los suelos característicos de la región, de llamativo color rojo, con óxidos de hierro y aluminio. Con frecuencia son asociados por mucha gente a la provincia de Misiones exclusivamente, pero también se encuentran en gran parte del nordeste correntino.

\section{Clima}

El clima es subtropical; en siete meses del año la temperatura media supera los $20^{\circ} \mathrm{C}$. El mes más cálido es enero, con $26.9^{\circ} \mathrm{C}$ de temperatura media, que fluctúa entre 15 y $16^{\circ} \mathrm{C}$ en los meses más fríos (junio-julio), en los que suelen producirse heladas. La precipitación anual es de aproximadamente $1700 \mathrm{~mm}$, con mayor concentración en meses de verano $(600 \mathrm{~mm})$, aunque ocasionalmente pueden producirse sequías estivales (estadísticas 1981-2010, Servicio Meteorológico Nacional 2016).

\section{Vegetación}

El nordeste de Corrientes se encuentra comprendido en el Distrito de los Campos de la Provincia Fitogeográfica Paranaense (Cabrera 1976). El mismo abarca unos $6000 \mathrm{~km} 2$ en el sur de la provincia de Misiones y $11177 \mathrm{~km} 2$ en la provincia de Corrientes (Carnevali 1994, Fontana 1996). Este Distrito también es denominado como "Ecorregión de Campos y Malezales" (Burkart et al. 1999).
Pajonales, pastizales y bosques constituyen la vegetación característica del área. Los bosques de la Provincia Paranaense ingresan hasta el nordeste de la provincia de Corrientes, donde alcanzan su límite austral, y configuran un paisaje único, con un conjunto de plantas y animales que no se encuentran en otras áreas de la misma. Estos bosques poseen su mayor superficie en Misiones, mientras que en Corrientes se hacen menos continuos y se presentan en isletas de variada extensión, rodeadas de pajonales y pastizales y formando "galerías" a lo largo de los arroyos y ríos de la región (Carnevali 1994).

Se pueden distinguir dos comunidades principales de bosque: en sectores con el basalto cercano a la superficie, crece el Bosque de urunday (Astronium balansae) y canela amarilla (Helietta apiculata), en tanto que en sitios con el suelo más profundo, crece el Bosque de anyico (Parapiptadenia rigida) y laurel negro (Nectandra megapotamica), similares en aspecto y composición florística a los del sur de Misiones (Eskuche 1986, Fontana 1996, 2015).

En la parte alta de las lomadas crecen pajonales mesófilos, sobre todo de flechilla (Aristida jubata) y paja colorada ( $A n-$ dropogon lateralis), en tanto que en sitios bajos o deprimidos, con encharcamiento semipermanente, se encuentran pajonales higrófilos de Andropogon virgatus (Eskuche 1986, Fontana 2015).

Los cultivos de yerba mate, té y maíz, y las forestaciones de pinos y eucaliptos constituyen las principales actividades productivas de la zona junto a la ganadería. 
Estos cultivos con frecuencia han reemplazado a los pajonales en las partes más altas de las lomadas, y actualmente constituyen un elemento característico del paisaje en el nordeste correntino.

\section{MÉTODOS}

De acuerdo a lo planificado, se efectuaron seis visitas al Sitio entre mayo y julio de 2015. Las mismas tuvieron como objetivo caracterizar la naturaleza del Sitio a través de:

1. Identificación de las especies vegetales más relevantes, por su abundancia, atractivo visual o por su relevancia para la fauna

2. Relevamientos de fauna, principalmente aves y mamíferos.

\section{Flora}

Las tareas de consistieron en la identificación de las especies de árboles y arbustos más llamativas a lo largo de los senderos, en base a guías de campo de la flora nativa (Fontana 2012, Soria et al. 2011, Peña-Chocarro et al. 2006). Se anotaron las especies más relevantes por su abundancia, atractivo visual o por su importancia para la fauna. Las especies fueron ordenadas según los estratos correspondientes a la estructura normal del bosque natural (Eskuche 1986).

\section{Fauna}

En el caso de la fauna del Sitio, sólo se consideraron aves y mamíferos. Las primeras fueron registradas tanto visualmente como a través de cantos y vocalizaciones durante recorridos de los senderos, sobre todo a primera hora de la mañana. También se incluyeron datos aportados por los guías intérpretes que se desempeñan en el Sitio, quienes suministraron fotografías y descripciones de varias especies. Cada especie fue asignada a una categoría de estacionalidad, basada en Mazar Barnett y Pearman (1998): Como especies Residentes, se consideraron a aquellas aves que son factibles de ser vitas en el predio del Sitio Arqueológico a diario, en cualquier estación del año, aunque no necesariamente nidifique allí. Como Migrantes Australes se consideraron a aquellas especies que nidifican en el área, no necesariamente en el Sitio, y pueden ser registrados allí durante la primavera y verano austral. Las especies Ocasionales fueron aquellas que si bien se encuentran presentes en el área, han sido registradas en el Sitio en una sola oportunidad sea por los investigadores o por los guías, y probablemente no formen parte habitual de la comunidad de aves.

La nomenclatura de las aves que se utilizó para este trabajo se basó en Remsen et al. (2016).

Los mamíferos presentes en el Sitio fueron registrados a través de avistajes directos, durante los recorridos de los senderos, y también a través de rastros (huellas, cuevas, fecas). También se contó con información brindada por los guías del Sitio. Tanto el caso de mamíferos y aves, sólo se consideraron para este trabajo aquellos datos de especies cuya identificación no planteaba dudas. 


\section{RESULTADOS}

\section{Vegetación}

La vegetación nativa del Sitio está constituida por un remanente de Bosque Paranaense (Fig. 3.A), rodeado por forestaciones de pinos (Pinus sp.), que poseen sotobosque formado por arbustos y arbolitos nativos. A pesar de la reducida superficie de esta formación vegetal en el Sitio Arqueológico, la misma tiene una estructura y composición similar a otras isletas de bosque del nordeste correntino y del sur misionero. Se pueden distinguir cuatro estratos bien diferenciados: arbóreo alto, arbóreo bajo, arbustivo y herbáceo. En ellos, se registraron 44 especies de árboles, arbustos, hierbas, trepadoras y epífitos (Tablas 1 y 2; Fig. 3.B a 3.F). De ellas, cuatro especies son exóticas (Hovenia dulcis, Carica papaya, Persea americana y Citrus reticulata).

La mayoría de las especies de árboles y arbustos del bosque producen frutos carnosos que constituyen un importante recurso para la fauna, ya que sus frutos son consumidos por una variedad de animales, desde aves hasta mamíferos como los carayás, coatíes, venados y murciélagos frugívoros. Incluso las cuatro especies exóticas asilvestradas que se registraron, probablemente han llegado a través de animales que se alimentaron de sus frutos y dispersaron sus semillas en el predio.

Dos especies de arbustos, Ruellia aff. sanguinea y Justicia brasiliana, poseen flores de color rojo y una estructura que denota que son ornitógamas, es decir, polinizadas por picaflores. Son muy conspicuos entre las especies del estrato arbustivo por su llamati- va floración y por el ir y venir de sus polinizadores que acuden a alimentarse del néctar.

Otra llamativa especie del estrato arbustivo, es el ñandipá-rá (Hennecartia omphalandra). A primera vista, parece poseer flores grandes de pétalos rosados, pero un examen más detenido, evidencia que las supuestas flores son en realidad sus frutos, que cuando maduros se abren y muestran su interior rojo con una gran semilla negra en el centro (Fig. 3.D).

El estrato herbáceo posee pocas especies: dos de pastos, cuatro de helechos, un subarbusto y una orquídea terrícola (Oeceoclades maculata), de pequeñas flores rosadas. Los helechos particularmente, cubren vastos sectores del suelo, sobre todo en sitios sombreados y húmedos.

Un elemento conspicuo en algunos sectores del bosque lo constituyen las lianas, debido a su profusión, al grosor que alcanzan y la forma en que se entrelazan, alcanzando las copas de los árboles más altos, donde despliegan su follaje y florecen. Una especie destacada entre ellas, es el isipó milhombres (Aristolochia triangularis), cuyas llamativas flores son polinizadas por moscas que ingresan a su interior, atraídas por el olor fétido de las mismas (Fig. 3.F).

La mayoría de los troncos están cubiertos por musgos epífitos, de formas y tamaños distintos, en algunos casos muy llamativos. Los epífitos vasculares son más bien escasos, y aparecen sólo en algunos árboles en puntos bien iluminados de los senderos. Se registraron una especie de helecho, dos de cactos y una especie de bromeliácea (Tabla 2). 

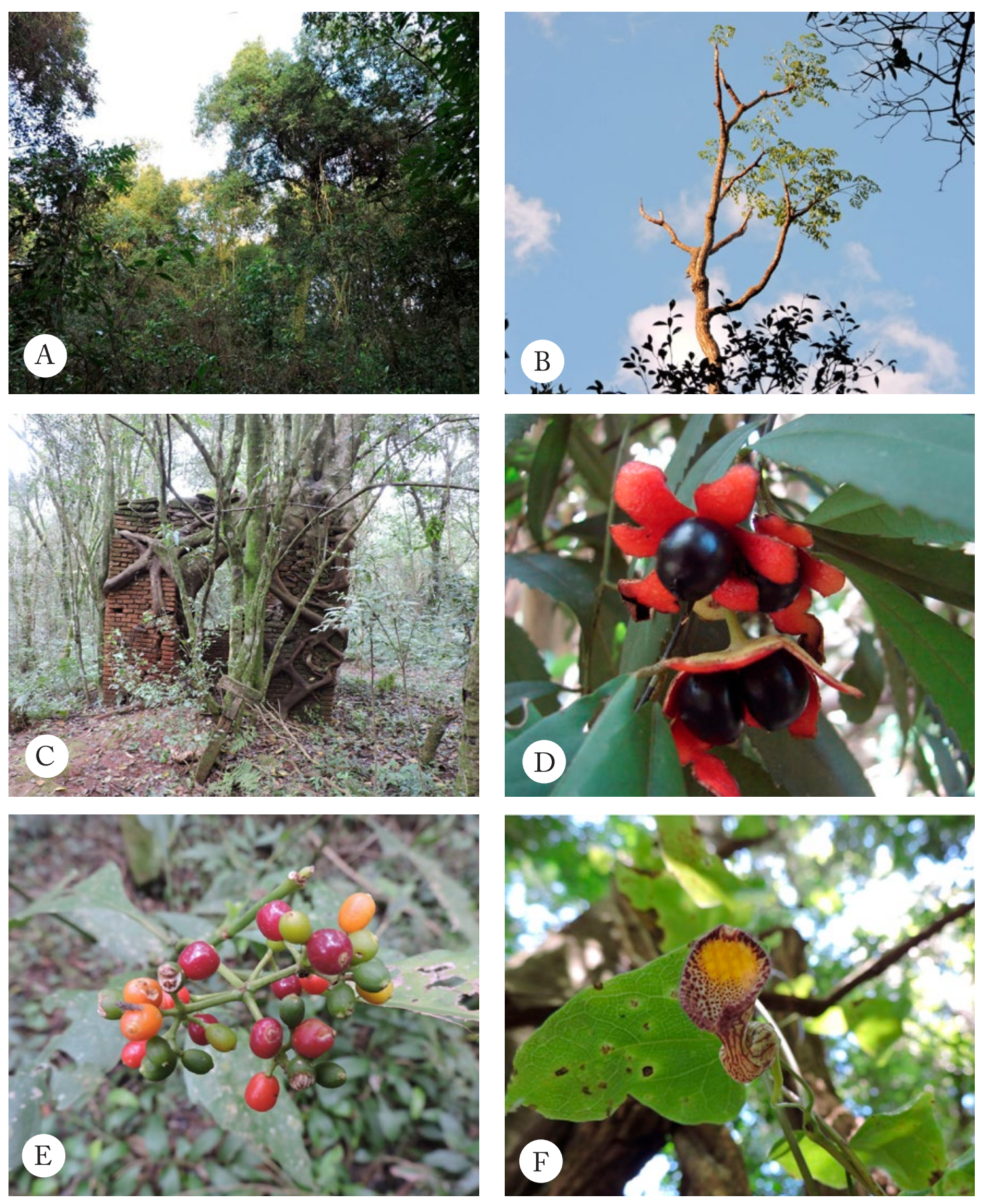

Fig. 3. A) Aspecto del bosque en el Sitio Arqueológico Capilla de San Alonso. B) Sabuguero (Aralia warmingiana), especie tipica del Bosque Parananense; C) Higuerón (Ficus luschnathiana), creciendo sobre una de las antiguas capillas del cementerio rural situado en el predio; D) Nandipá-rá (Hennecartia omphalandra); E) Psychotria carthagenensis; F) Isipó milhombres (Aristolochia triangularis), liana muy utilizada en la medicina popular. 
Tabla 1. Especies vegetales identificadas en el Sitio Arqueológico, ordenadas por estratos. Entre paréntesis, se indica la altura promedio de cada uno de los estratos. Con "+" se señalan las especies exóticas asilvestradas en este rodal.

\begin{tabular}{|c|c|}
\hline Especies por estrato & Nombre científico \\
\hline \multicolumn{2}{|l|}{ Estrato Arbóreo Alto $( \pm 16 \mathrm{~m})$} \\
\hline $\begin{array}{l}\text { Laurel Negro } \\
\text { Ingá pytá } \\
\text { Sabuguero } \\
\text { Higuerón } \\
\text { Ubeña }^{+} \\
\text {Pindó } \\
\text { Mbocayá } \\
\text { Ambay } \\
\text { Guabiyú }\end{array}$ & $\begin{array}{l}\text { Nectandra megapotamica } \\
\text { Inga urugüensis } \\
\text { Aralia warmingiana } \\
\text { Ficus luschnathiana } \\
\text { Hovenia dulcis } \\
\text { Syagrus romanzoffiana } \\
\text { Acrocomia aculeata } \\
\text { Cecropia pachystachya } \\
\text { Myrcianthes pungens } \\
\end{array}$ \\
\hline \multicolumn{2}{|l|}{ Estrato Arbóreo Alto (8-10 m) } \\
\hline $\begin{array}{l}\text { Camboatá } \\
\text { Arachichú } \\
\text { Canela de venado } \\
\text { Nandipá-rá } \\
\text { Cuero de vieja } \\
\text { Mamica de cadela, Teta de perra } \\
\text { Mamón }^{+} \\
\text {Palta }^{+}\end{array}$ & $\begin{array}{l}\text { Cupania vernalis } \\
\text { Rollinia emarginata } \\
\text { Helietta apiculata } \\
\text { Hennecartia omphalandra } \\
\text { Guarea macrophylla } \\
\text { Zanthoxylum rhoifolium } \\
\text { Carica papaya } \\
\text { Persea americana } \\
\end{array}$ \\
\hline \multicolumn{2}{|l|}{ Estrato Arbustivo (4 m) } \\
\hline $\begin{array}{l}\text { Cedrillo } \\
\text { Pitanga } \\
-- \\
\text { Coralito } \\
-- \\
\text { Varana } \\
\text { Yerba mate } \\
\text { Vasuriña } \\
-- \\
\text { Mandarino } \\
\text { Cocú }\end{array}$ & $\begin{array}{l}\text { Trichilia elegans } \\
\text { Eugenia uniflora } \\
\text { Piper sp. } \\
\text { Justicia brasiliana } \\
\text { Ruellia aff. sanguinea } \\
\text { Cordyline sellowiana } \\
\text { Ilex paraguariensis } \\
\text { Chrysophyllum gonocarpum } \\
\text { Psychotria carthagenensis } \\
\text { Citrus reticulata } \\
\text { Allophylus edulis } \\
\end{array}$ \\
\hline \multicolumn{2}{|l|}{ Estrato Herbáceo (1 m) } \\
\hline $\begin{array}{l}\text { Pasto } \\
\text { Pasto } \\
\text { Helecho } \\
\text { Helecho sp. } 1 \\
\text { Helecho sp. } 2 \\
\text { Helecho sp. } 3 \\
\text { Orquídea terrícola } \\
\text {-- }\end{array}$ & $\begin{array}{l}\text { Oplismenus hirtellus } \\
\text { Cf. Pharus glaber } \\
\text { Cheilanthes concolor } \\
-- \\
-- \\
-- \\
\text { Oeceoclades maculata } \\
\text { Pavonia malvaceal }\end{array}$ \\
\hline
\end{tabular}


Tabla 2. Especies de lianas y epifitos registrados en el bosque del Sitio Arqueológico.

\begin{tabular}{ll}
\hline Trepadoras & Nombre científico \\
\hline Isipó milhombres & Aristolochia triangularis \\
Mburucuyá & Passiflora elegans \\
-- & Serjania sp. \\
Fabaceae no identificada & -- \\
\hline Epífitos & Nombre científico \\
\hline Cacto epífito & Epiphyllum phyllanthus \\
Suelda con suelda, cacto epífito & Rhipsalis sp. \\
Caraguatá & Aechmea bromeliifolia \\
Helecho epífito & Microgramma vacciniifolia \\
& \\
\hline
\end{tabular}

Aves

Se registraron 65 especies de aves, pertenecientes a 28 familias (Tabla 3). El número es elevado, para un área de reducida superficie como es el predio donde se encuentra la Capilla Jesuítica. Varias de las especies son características de la Provincia Paranaense, que comprende en nuestro país a la provincia de Misiones y nordeste de Corrientes, no hallándose presentes en el resto del territorio de esta última; entre estas:

1. Carpintero Oliva Manchado (Veniliornis spilogaster)

2. Yacutoro (Pyroderus scutatus)

3. Saí Azul (Dacnis cayana)

4. Saíra Pecho Negro (Tangara cayana)

5. Frutero Corona Amarilla (Trichothraupis melanops)

6. Frutero Coronado (Tachyphonus coronatus)

Cincuenta y cuatro de las especies de aves registradas (83\%) se consideran residentes en el Sitio; seis especies (9\%)
(Myiodynastes maculatus, Empidonomus varius, Tyrannus melancholicus, Megarbynchus pitangua y Tyrannus savana) son visitantes estivales, presentes en la región en primavera-verano, migrando al norte con la llegada del otoño; dos (3\%) (Buteo brachyurus y Pyroderus scutatus) se consideran como ocasionales en el Sitio, aunque están presentes en el área, y de tres (5\%) (una especie no identificada de Caprimulgidae, Dacnis cayana y Tangara cayana) no se conoce con certeza si son residentes (Tabla 3)

Aunque no se han detectado aves amenazadas o en peligro de extinción, el conjunto de especies registrado en el Sitio posee gran valor para el área y es un importante atractivo turístico: entre las especies más llamativas por su tamaño, colorido o vocalizaciones, se encuentran los tucanes (Ramphastos toco), yacúes o pavas de monte (Penelope obscura), surucuás (Trogon surrucura) y boyeros caciques (Cacicus haemorrhous). Otras especies de presencia menos frecuente, pero de gran valor y atractivo 
para potenciales observadores de aves son ra cayana, respectivamente), los yacú toro los zorzales (Turdus spp., Fig. 4.B), saíes y (Pyroderus scutatus) y los llamativos yapúes saíras (Dacnis cayana [Fig. 4.C] y Tanga- (Psarocolius decumanus) (Fig. 4.D).

Tabla 3. Lista de las especies de aves registradas en el Sitio Arqueológico Capilla de San Alonso. R: Especie residente; MA: Migrante Austral; O: Especie ocasional en el Sitio; ?: Categoría dudosa.

\begin{tabular}{|c|c|c|}
\hline NOMBRE COMÚN & NOMBRE CIENTÍFICO & ESTACIONALIDAD \\
\hline \multicolumn{3}{|l|}{ Familia Tinamidae } \\
\hline Tataupá Común, Perdiz Blanca & Crypturellus tataupa & $R$ \\
\hline \multicolumn{3}{|l|}{ Familia Cathartidae } \\
\hline Cuervo de Cabeza Amarilla & Cathartes burrovianus & $R$ \\
\hline Cuervo de Cabeza Negra & Coragyps atratus & $R$ \\
\hline \multicolumn{3}{|l|}{ Familia Accipitridae } \\
\hline Aguilucho Cola Corta & Buteo brachyurus & $O$ \\
\hline Taguató Común & Rupornis magnirostris & $R$ \\
\hline \multicolumn{3}{|l|}{ Familia Falconidae } \\
\hline Chimachima & Milvago chimachima & $R$ \\
\hline Halconcito Colorado & Falco sparverius & $R$ \\
\hline \multicolumn{3}{|l|}{ Familia Cracidae } \\
\hline Yacú, Pava de Monte & Penelope obscura & $R$ \\
\hline \multicolumn{3}{|l|}{ Familia Rallidae } \\
\hline Pacaá & Aramides ypecaha & $R$ \\
\hline Syrykó, Chiricote & Aramides cajanea & $R$ \\
\hline \multicolumn{3}{|l|}{ Familia Columbidae } \\
\hline Paloma Picazuró & Patagioenas picazuro & $R$ \\
\hline Yerutí & Leptotila verreauxi & $R$ \\
\hline Yerutí Colorada & Leptotila rufaxilla & $R$ \\
\hline Torcaza & Zenaida auriculata & $R$ \\
\hline Torcacita, Picuí & Columbina picui & $R$ \\
\hline Torcacita Colorada & Columbina talpacoti & $R$ \\
\hline \multicolumn{3}{|l|}{ Familia Cuculidae } \\
\hline Alma de Gato & Piaya cayana & $R$ \\
\hline \multicolumn{3}{|l|}{ Familia Strigidae } \\
\hline Araracucú & Megascops choliba & $R$ \\
\hline \multicolumn{3}{|l|}{ Familia Nyctibiidae } \\
\hline Urutaú & Nyctibius griseus & $M A$ \\
\hline \multicolumn{3}{|l|}{ Familia Caprimulgidae } \\
\hline Especie no identificada & -- & ? \\
\hline \multicolumn{3}{|l|}{ Familia Trochilidae } \\
\hline Picaflor Bronceado & Hylocharis chrysura & $R$ \\
\hline Picaflor Común & Chlorostilbon lucidus & $R$ \\
\hline \multicolumn{3}{|l|}{ Familia Trogonidae } \\
\hline Surucuá Común & Trogon surrucura & $R$ \\
\hline \multicolumn{3}{|l|}{ Familia Ramphastidae } \\
\hline Tucán Grande & Ramphastos toco & $R$ \\
\hline \multicolumn{3}{|l|}{ Familia Picidae } \\
\hline Carpintero Blanco & Melanerpes candidus & $R$ \\
\hline Carpintero Oliva Manchado & Veniliornis spilogaster & $R$ \\
\hline
\end{tabular}




\begin{tabular}{|c|c|c|}
\hline Carpinterito & Picumnus sp. & $\begin{array}{l}R \\
R\end{array}$ \\
\hline Carpintero Garganta Estriada & & \\
\hline \multicolumn{3}{|l|}{ Familia Furnariidae } \\
\hline Alonsito. Hornero & Furnarius rufus & $R$ \\
\hline Pijuí Frente Gris & Synallaxis frontalis & $R$ \\
\hline \multicolumn{3}{|l|}{ Familia Thamnophilidae } \\
\hline Choca Común & Thamnophilus caerulescens & $R$ \\
\hline \multicolumn{3}{|l|}{ Familia Tyrannidae } \\
\hline Pitogüé Rayado & Myiodynastes maculatus & MA \\
\hline Tuquito Rayado & Empidonomus varius & \\
\hline Pitogüé & Pitangus sulphuratus & $R$ \\
\hline Picabuey & Machetornis rixosa & $R$ \\
\hline Suirirí Real & Tyrannus melancholicus & MA \\
\hline Tijereta & Tyrannus savana & MA \\
\hline Pitanguá & Megarhynchus pitangua & MA \\
\hline \multicolumn{3}{|l|}{ Familia Cotingidae } \\
\hline Yacutoro & Pyroderus scutatus & $O$ \\
\hline \multicolumn{3}{|l|}{ Familia Vireonidae } \\
\hline Juan Chiviro & Cyclarhis gujanensis & $R$ \\
\hline \multicolumn{3}{|l|}{ Familia Corvidae } \\
\hline Urraca Común & Cyanocorax chrysops & $R$ \\
\hline \multicolumn{3}{|l|}{ Familia Troglodytidae } \\
\hline Tacuarita & Troglodytes aedon & $R$ \\
\hline \multicolumn{3}{|l|}{ Familia Turdidae } \\
\hline Zorzal Chalchalero & Turdus amaurochalinus & $R$ \\
\hline Zorzal Sabiá & Turdus leucomelas & $R$ \\
\hline Zorzal Colorado & Turdus rufiventris & $R$ \\
\hline Zorzal Collar Blanco & Turdus albicollis & $R$ \\
\hline \multicolumn{3}{|l|}{ Familia Parulidae } \\
\hline Arañero Silbón & Basileuterus leucoblepharus & $R$ \\
\hline Arañero Coronado Chico & Basileuterus culicivorus & $R$ \\
\hline Pitiayumi & Parula pitiayumi & $R$ \\
\hline \multicolumn{3}{|l|}{ Familia Thraupidae } \\
\hline Saí Azul & Dacnis cayana & $R$ ? \\
\hline Tangará Común & Euphonia chlorotica & $R$ \\
\hline Saíra Pecho Negro & Tangara cayana & $R$ ? \\
\hline Chogüí & Thraupis sayaca & $R$ \\
\hline Fueguero Común & Piranga flava & $R$ \\
\hline Frutero Coronado & Tachyphonus coronatus & $R$ \\
\hline Frutero Corona Amarilla & Trichothraupis melanops & $R$ \\
\hline \multicolumn{3}{|l|}{ Familia Emberizidae } \\
\hline Chingolo & Zonotrichia capensis & $R$ \\
\hline \multicolumn{3}{|l|}{ Familia Cardinalidae } \\
\hline Fueguero Común & Piranga flava & $R$ \\
\hline Reinamora Grande & Cyanocompsa brissonii & $R$ \\
\hline Pepitero Verdoso & Saltator similis & $R$ \\
\hline \multicolumn{3}{|l|}{ Familia Icteridae } \\
\hline Yapú & Psarocolius decumanus & $R$ \\
\hline Boyero Ala Amarilla & Cacicus chrysopterus & $R$ \\
\hline Boyero Negro & Cacicus solitarius & $R$ \\
\hline Boyero Cacique & Cacicus haemorrhous & $R$ \\
\hline Boyerito & Icterus cayanensis & $R$ \\
\hline
\end{tabular}




\section{Mamíferos}

Ocho especies de mamíferos, pertenecientes a seis familias fueron detectados en el Sitio (Tabla 4), a través de avistajes directos, presencia de cuevas, huellas, y también encuestas efectuadas a los guía intérpretes que desarrollan sus actividades en el lugar. Si bien parece una cifra muy reducida si se compara con el número de especies de aves, debe tenerse en cuenta que los mamíferos registrados son de mediano
La "poca" (Mazama nana) es una especie de venado de pequeño porte, característica de la Provincia Paranaense, registrada a través de las encuestas y registro de huellas. Reviste particular importancia por ser una especie considerada "Vulnerable" en nuestro país (Lartigau et al. 2012).

Los coatíes (Nasua nasua) (Fig. 4.E) son los mamíferos más carismáticos del lugar, debido a su aspecto y sus hábitos grupales. Tienen gran importancia para

Tabla 4. Lista de mamíferos registrados en el Sitio Arqueológico

\begin{tabular}{ll} 
Nombre Común & Nombre científico \\
\hline $\begin{array}{l}\text { Familia Dasypodidae } \\
\text { Tatú Negro }\end{array}$ & $\begin{array}{l}\text { Dasypus novemcinctus } \\
\text { Euphractus sexcinctus }\end{array}$ \\
\hline $\begin{array}{l}\text { Tatú Peludo } \\
\text { Camilia Atelidae }\end{array}$ & Alouatta caraya \\
\hline $\begin{array}{l}\text { Familia Canidae } \\
\text { Aguará-í, Zorro de Monte }\end{array}$ & Cerdocyon thous \\
\hline $\begin{array}{l}\text { Familia Felidae } \\
\text { Especie no identificada }\end{array}$ & -- \\
\hline $\begin{array}{l}\text { Familia Procyonidae } \\
\text { Coatí }\end{array}$ & Nasua nasua \\
\hline $\begin{array}{l}\text { Familia Cervidae } \\
\text { Guazuncho, Venado } \\
\text { Poca }\end{array}$ & Mazama gouazoubira \\
\hline
\end{tabular}

tamaño, con masas corporales que van desde 2 a más de $20 \mathrm{~kg}$, como los venados, y otros de hábitos sociales como los coatíes, que se desplazan en grupos que superan los 30 individuos. Aunque estas especies no siempre pueden ser observadas en el Sitio, dados sus amplios desplazamientos territoriales, que incluyen las forestaciones con sotobosque de las inmediaciones, tampoco resultan raros de ver o detectar a través de las señales que dejan (huellas, excrementos). el Sitio, debido a que es uno de los pocos lugares del nordeste de Corrientes donde se encuentran. Debido a esto, fue la especie seleccionada para estar presente en la cartelería de las paradas de interpretación biológica.

Otros mamíferos que se presentan en grupo, muy llamativos para los visitantes, son los carayás (Alouatta caraya) (Fig. 4.F), que llegan hasta el Sitio desplazándose a través de las forestaciones con sotobosque que rodean al Sitio. Junto a los coatíes, son 

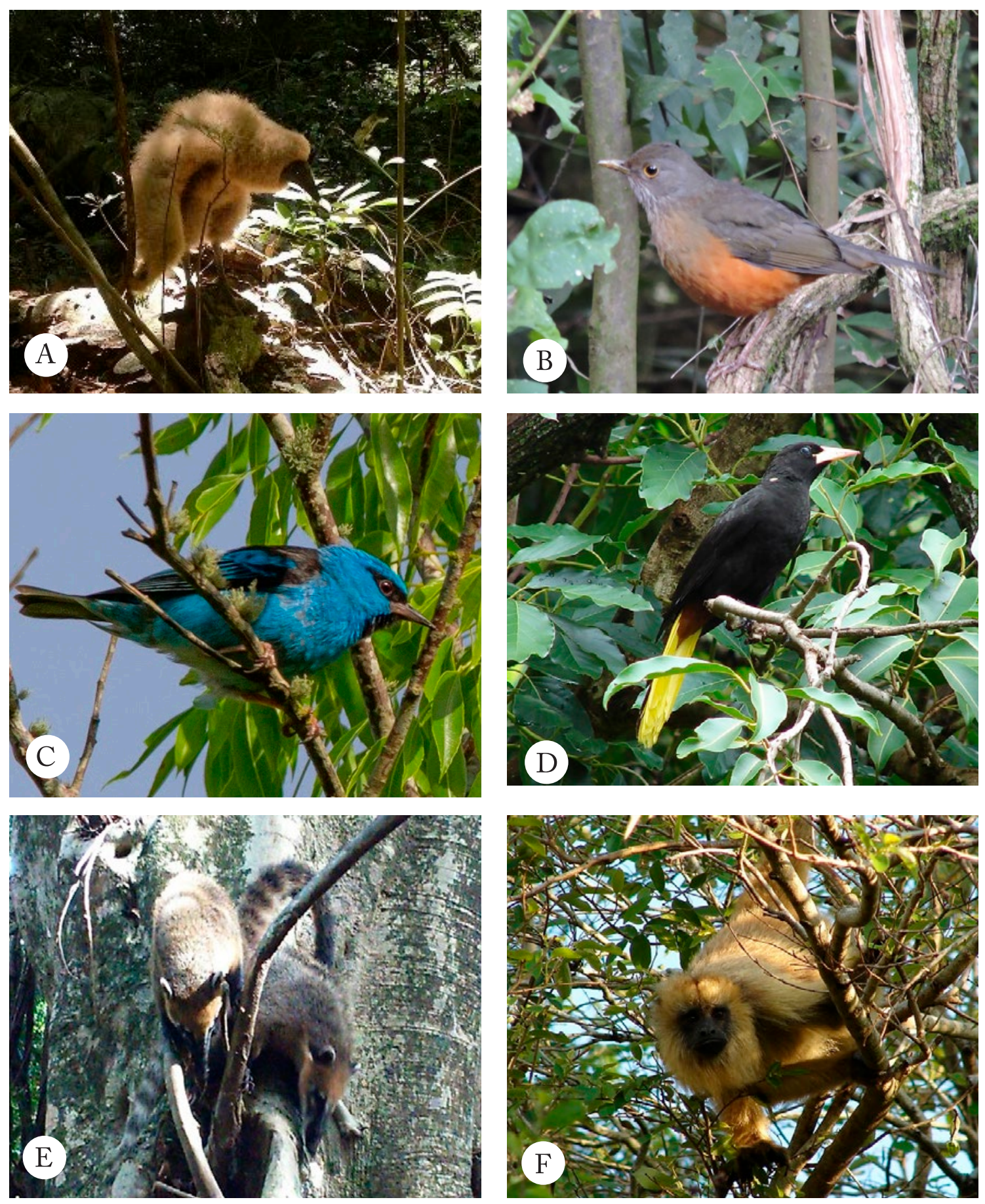

Fig. 4.A) Pichón de Cuervo de Cabeza Negra (Coragyps atratus), especie registrada nidificando en el Sitio (Foto: Valeria Sequeira; B) Zorzal Colorado (Turdus rufiventris); C) Sai Azul (Dacnis cayana) (Foto: Sebastián Navajas); D) Yapú (Psarocolius decumanus). E) Coaties (Nasua nasua) (Foto: Valeria Sequeira); F) Carayá hembra (Alouatta caraya). 
dispersores de semillas de varias de las especies de árboles y arbustos.

Sería importante en una próxima etapa, realizar un relevamiento que incluya a roedores, pequeños marsupiales y murciélagos, grupos poco estudiados en el área y que podrían brindar registros novedosos.

\section{CONSIDERACIONES FINALES}

Sitio Arqueológico Capilla de San Alonso posee una muestra de la biodiversidad del nordeste correntino, a pesar de su reducida superficie. Aunque se dispone de inventarios parciales de plantas y animales, los mismos aún deberían completarse con relevamientos de otros grupos como los insectos, particularmente las mariposas, cuya diversidad de especies, tamaños y colores las vuelven muy conspicuas y llamativas a los visitantes.

$\mathrm{Su}$ flora y su fauna constituyen un atractivo adicional a las ruinas jesuíticas, que despierta el interés de los turistas que visitan el Sitio. Además del turismo, brinda excelentes oportunidades para la educación, la investigación y la capacitación. Esto constituye un argumento de peso para la conservación de su naturaleza de la manera menos modificada posible, para garantizar su permanencia a lo largo del tiempo.

\section{AGRADECIMIENTOS}

A la Municipalidad y a la Dirección de Turismo de Gobernador Virasoro, por su apoyo para la concreción de este proyecto, y en particular a los guías intérpretes del Sitio: Nicolás Kohan, César Fernández y Ricardo Arévalo. A Valeria Sequeira y Sebastián Navajas, por poner a disposición algunas de las fotos que ilustran este trabajo. A José Radins, por la identificación de algunas de las plantas del Sitio.

\section{BIBLIOGRAFÍA}

BURKART, R.; N. O. BÁRBARO, R. O. fía de la Provincia de Corrientes. EdiSÁNCHEZ y D. A. GÓMEZ. 1999. Eco-regiones de la Argentina. Administración de Parques Nacionales, Buenos Aires.

CABRERA A. L. 1976. Regiones fitogeográficas argentinas. Enciclopedia Argentina de Agricultura y Jardinería II. 1.2 a ed. ACME, Buenos Aires. CARNEVALI R. 1994. Fitogeogratora Litocolor S.A., Corrientes. ESKUCHE, U. 1986. Relación sobre la 17a Excursión Fitogeográfica Internacional por la Argentina Septentrional. En: U. ESKUCHE y E. LANDOLT (Eds.), Contribuciones al conocimiento de flora y vegetación del norte de la Argentina. Veröff. Geobot. Inst. ETH, Stiftung. Rübel in Zürich 91:12-177. 
FONTANA, J. L. 1996. Los pajonales mesófilos semi-naturales de Misiones (Argentina). Phytocoenologia 26: 179-271. FONTANA, J. L. 2012. Guía de plantas. Reserva Natural Isla Apipé Grande, provincia de Corrientes, Argentina. $2^{\circ}$ edic. ampliada. Edición del Autor. Corrientes. FONTANA, J. L. 2015. Capítulo 1. Flora y vegetación del nordeste de Corrientes y sur de Misiones. Pp. 9-27. En: V. BAUNI, M. A. HOMBERG y V. CAPMOURTERES (Eds.) El patrimonio natural y cultural en el área de influencia del embalse de Yacyretá, Argentina. Fundación de Historia Natural Félix de Azara, Buenos Aires. LARTIGAU, B., C. DE ANGELO, S. D’ALESSIO, I. JIMÉNEZ PÉREZ, G. APRILE, M. B. AUED, N. FRACASSI y D. VARELA. 2012. Familia Cervidae. Pp. 121-128. En: R. A. OJEDA, V. CHILLO y G. B. DÍAZ ISENRATH (Eds.). Libro Rojo de Mamíferos Amenazados de Argentina. SAREM, Tucumán. MAZAR BARNETT, J. Y PEARMAN, M. 2001. Lista comentada de las aves argentinas. Lynx Ediciones, Barcelona, PEÑA-CHOCARRO, M. C., J. DE EGEA JUVINEL, M. VERA, H. MATURO y S. KNAPP. 2006. Guía de árbo- les y arbustos del Chaco Húmedo. The Natural History Museum, Guyrá Paraguay, Fundación Moisés Bertoni y Fundación Hábitat y Desarrollo. Asunción. POPOLIZIO, E. 1989. Algunos elementos geomorfológicos condicionantes de la organización espacial y las actividades del NEA. Geociencias, 17: 3-12. REMSEN, J. V., JR., J. I. ARETA, C. D. CADENA, A. JARAMILLO, M. NORES, J. F. PACHECO, J. PÉREZ-EMÁN, M. B. ROBBINS, F. G. STILES, D. F. STOTZ y K. J. ZIMMER. 2016. A classification of the bird species of South America. American Ornithologists' Union. http:// www.museum.lsu.edu/ Remsen/SACCBaseline.html. Versión Febrero de 2016. SERVICIO METEOROLÓGICO NACIONAL. 2016. Atlas climático periodo 1981-2010. http:// www.smn.gov.ar/serviciosclimaticos/ SORIA, A., J. C. CHEBEZ, S. F. FABRI y C. M. GONZÁLEZ. 2011. Misiones. Árboles/Trees/Árbores. Golden Company. Buenos Aires. TERUGGI, M. E. 1970. Bosquejo geológico del Paraguay y la provincia de $\mathrm{Co}^{-}$ rrientes. Boletín de la Sociedad Argentina de Botánica, 11 (Suplemento): 1 - 17. 